\title{
Understanding CH-Stretching Raman Optical Activity in Ala-Ala Dipeptides
} Marius Hope, ${ }^{\dagger+}$ Jaroslav Šebestík, ${ }^{\dagger}$ Josef Kapitán, ${ }^{\S}$ and Petr Bouř ${ }^{\dagger}$

$\dagger$ Institute of Organic Chemistry and Biochemistry, Academy of Sciences, Flemingovo námèstí 2, 16610 Prague, Czech Republic.

* Norwegian University of Science and TechnologyNO-7491 Trondheim, Norway.

${ }^{\S}$ Department of Optics, Palacký University, 17. listopadu 12, 77146 Olomouc, Czech Republic.

\section{Contents}

Figure S1. Experimental ROA and Raman spectra, full range.

Figure S2. Raw Raman spectra of protonated and deprotonated Ala-Ala.

Figure S3. Calculated spectra, functional dependence.

Figure S4. Calculated spectra, basis set dependence.

Figure S5. Protonated Ala-Ala, CH stretching Raman and ROA spectra, MD vs. PCM.

Figure S6. Deprotonated Ala-Ala, CH stretching Raman and ROA spectra, MD vs. PCM.

Figure S7. Ala-Ala zwitterion, spectra calculated with various water models.

Figure S8. Comparison of the harmonic and anharmonic/PT2 calculations.

Figure S9. Comparison of the harmonic and anharmonic/LVCI calculations.

Table S1. Main Experimental Bands and their ROA/Raman ratios

Table S2. Principal Torsion Angles

Table S3. AlaAla Vibrational Fundamental Modes 


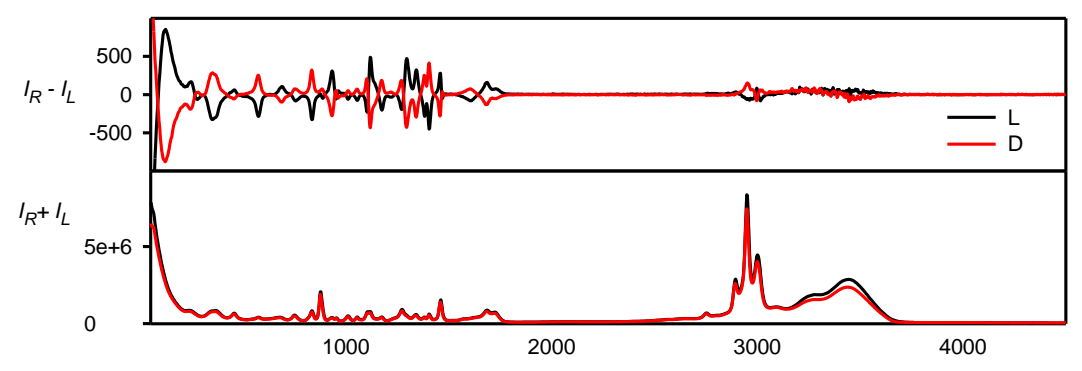

Neutral Ala-Ala

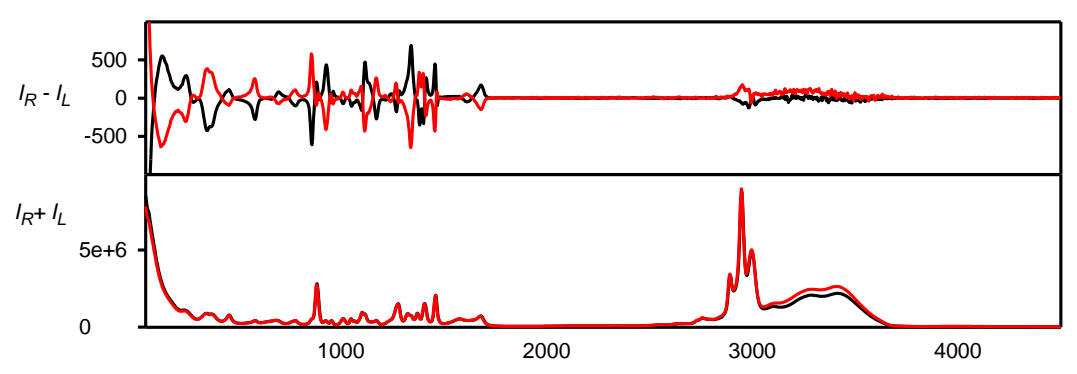

Deprotonated Ala-Ala

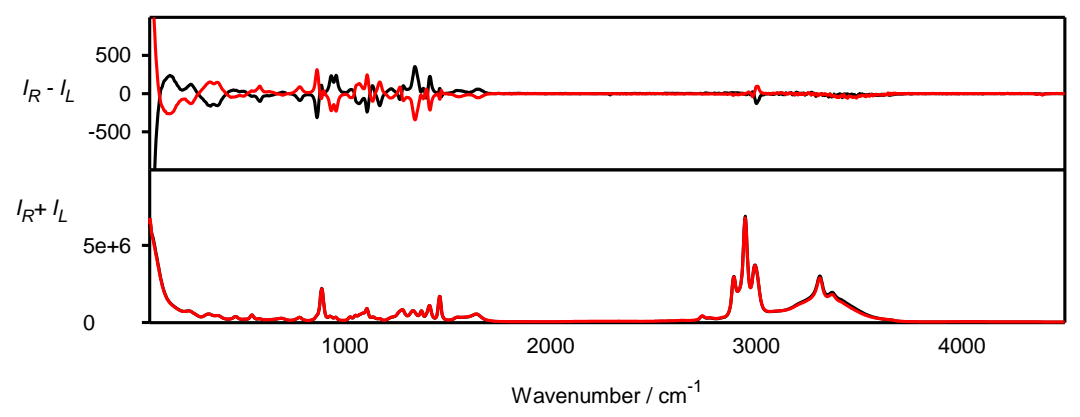

Figure S1. SCP ROA $\left(I_{R}-I_{L}\right)$ and Raman $\left(I_{R}+I_{L}\right)$ spectra of the three Ala-Ala forms shown in the full range of the spectrometer $\left(50 \ldots 4500 \mathrm{~cm}^{-1}\right.$, measured at $\mathrm{pH} 1, \mathrm{pH} 7$ and $\mathrm{pH} 13$, respectively). 


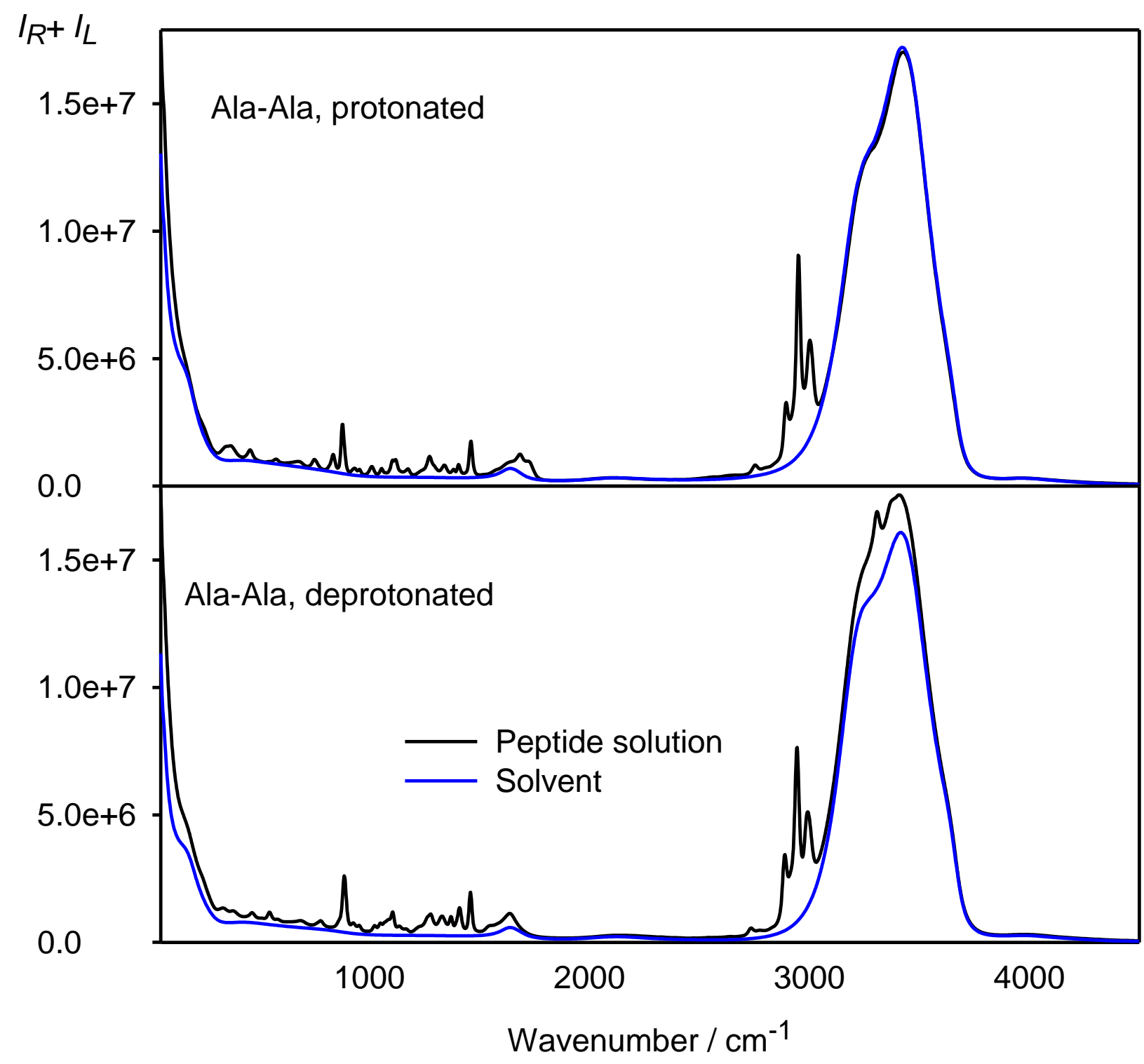

Figure S2. Raw Raman spectra of protonated ( $\mathrm{pH}$ 1) and deprotonated (pH 13) Ala-Ala solutions, and the $\mathrm{HCl}$ and $\mathrm{NaOH}$ water solutions. 


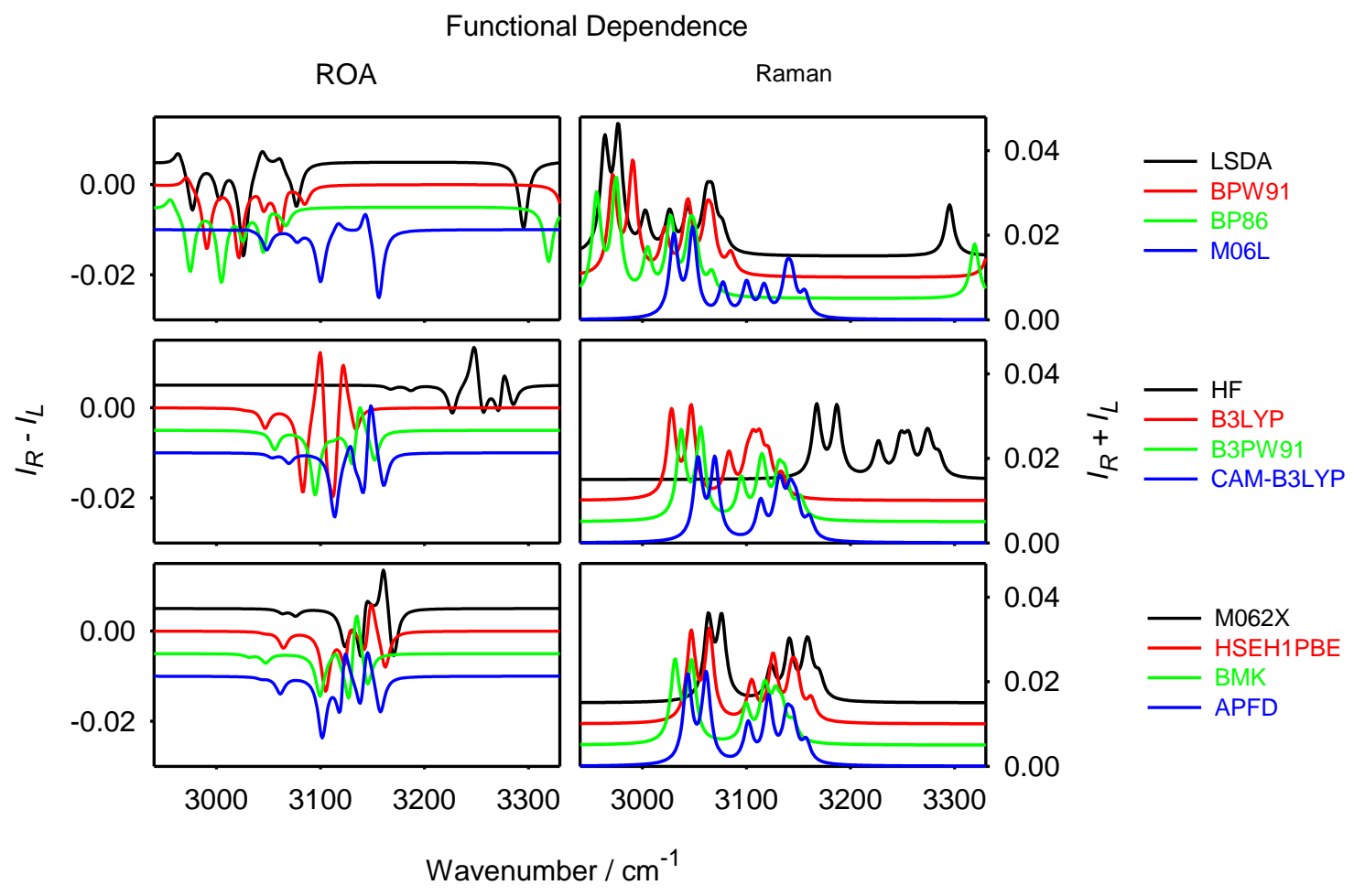

Figure S3. ROA and Raman spectra of Ala-Ala zwitterion calculated with 12 functionals (6$311++\mathrm{G}^{* *} / \mathrm{PCM} / \mathrm{H}_{2} \mathrm{O}$, some spectra are $y$-offset for better visibility). The LSDA and GGA ("pure") functionals comprise the local spin density approximation, ${ }^{1}$ Becke $^{2}$ exchange with the Perdew/Wang ${ }^{3}$ or Perdew ${ }^{4}$ correlation in BPW91 and BP86, and M06L. ${ }^{5}$ Hartee-Fock (HF) and B3LYP, ${ }^{6}$ B3PW91 $1^{3,6}$ and CAM-B3LYP ${ }^{7}$ hybrid functionals are in the middle. The bottom panel comprises newer M062X, ${ }^{8} \mathrm{HSEH}_{1 \mathrm{PBE}}{ }^{9} \mathrm{BMK}^{10}$ and dispersion-containing APFD. ${ }^{11}$ Similar results were obtained using the MP2 $2^{12}$ force field and B3LYP tensor derivatives. 


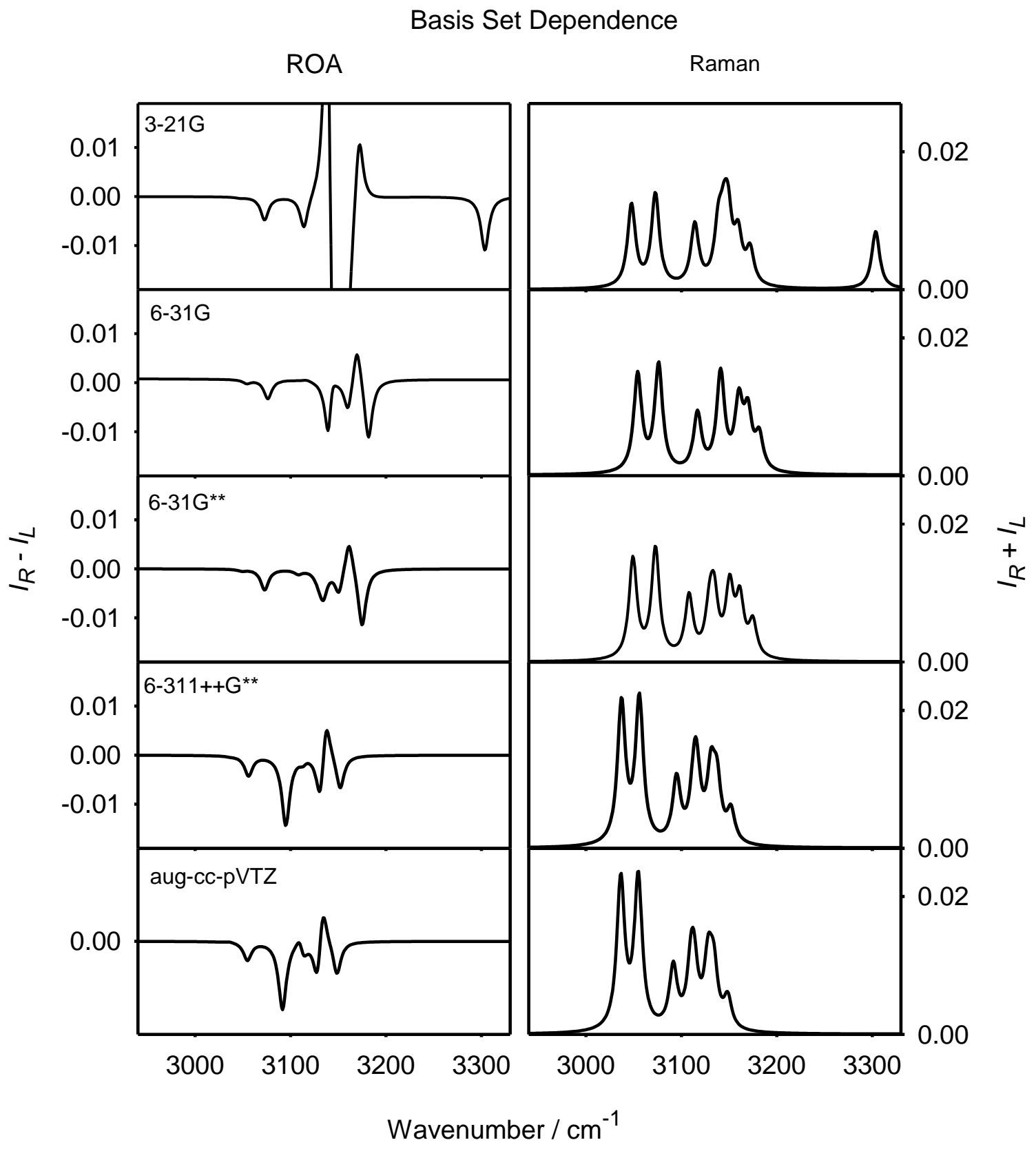

Figure S4. Ala-Ala zwitterion, ROA and Raman spectra calculated with various basis sets and the B3PW91/PCM model. 


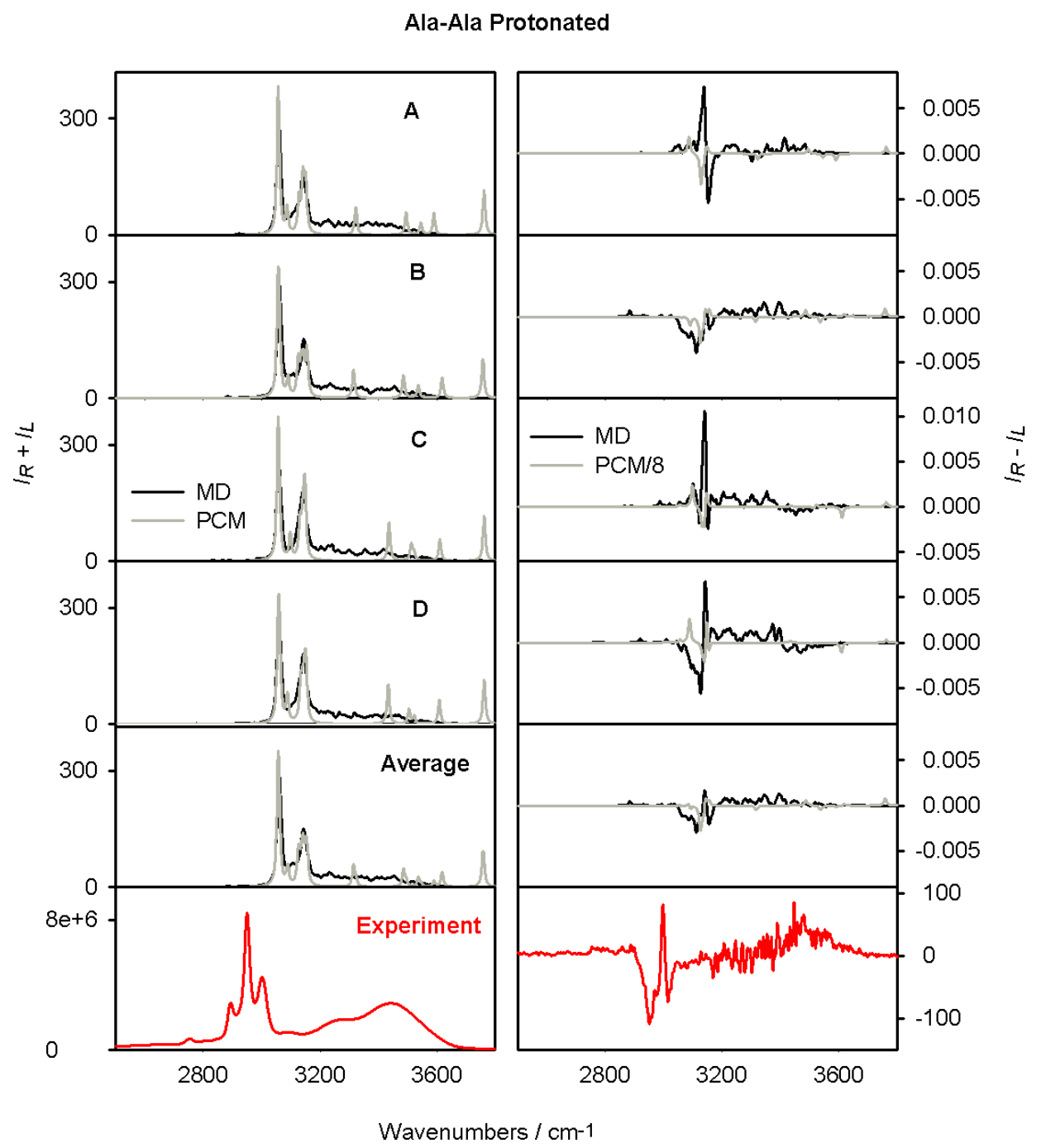

Figure S5. Protonated Ala-Ala, simulated Raman and ROA spectra of the four conformers (A-D), Boltzmann average, and the experiment. The simulations were performed at the B3PW91/6$311++\mathrm{G}^{* *} / \mathrm{PCM}$ level for the optimized geometry ("PCM") and on $100 \mathrm{MD}$ snapshots for each conformer (MD). The PCM ROA spectra were divided by 8 . 


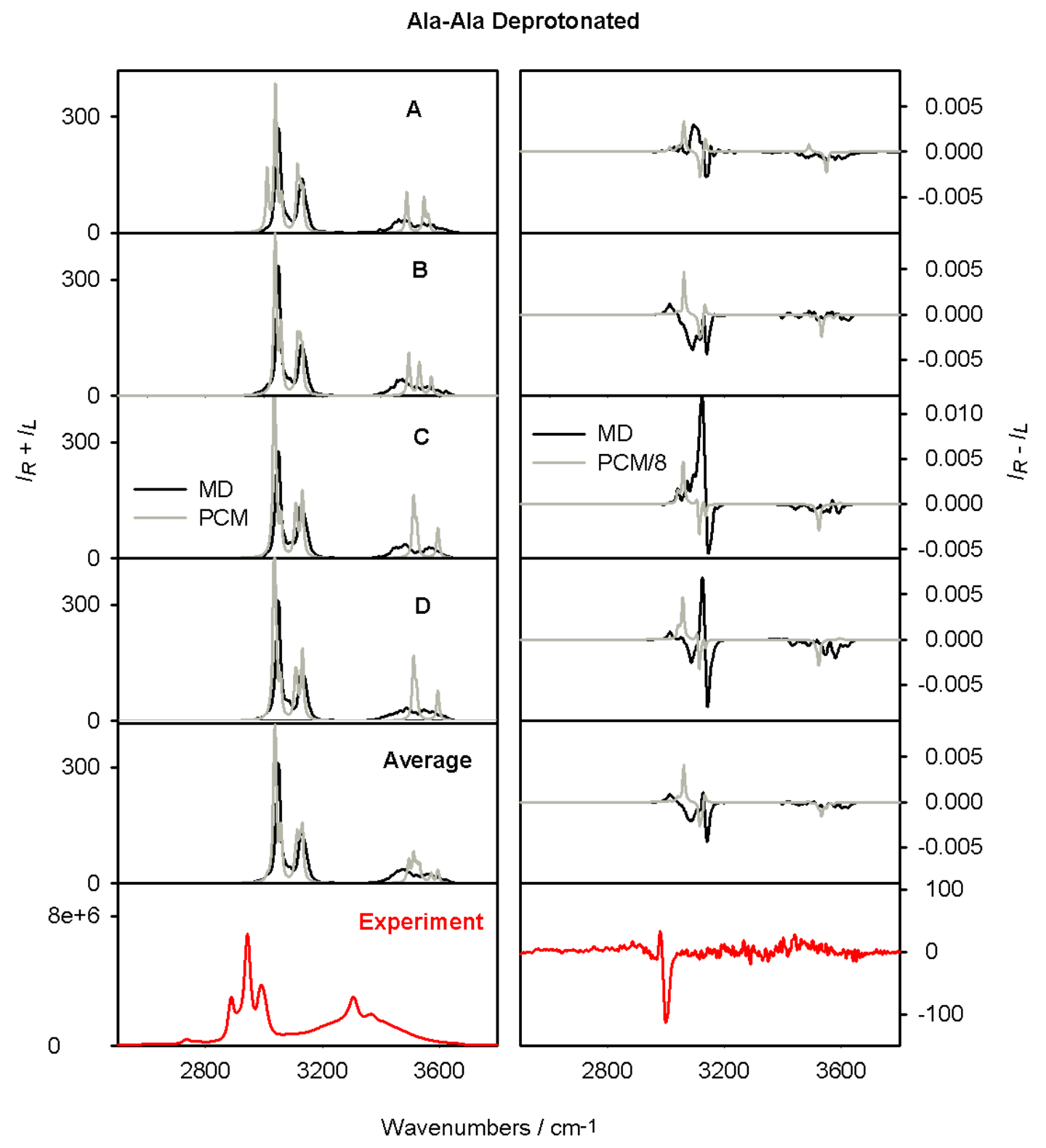

Figure S6. Deprotonated Ala-Ala, simulated Raman and ROA spectra of the four conformers (AD), Boltzmann average, and the experiment. The simulations were performed at the B3PW91/6$311++\mathrm{G}^{* *} / \mathrm{PCM}$ level for the optimized geometry ("PCM") and on $100 \mathrm{MD}$ snapshots for each conformer (MD). The PCM ROA spectra were divided by 8 . 

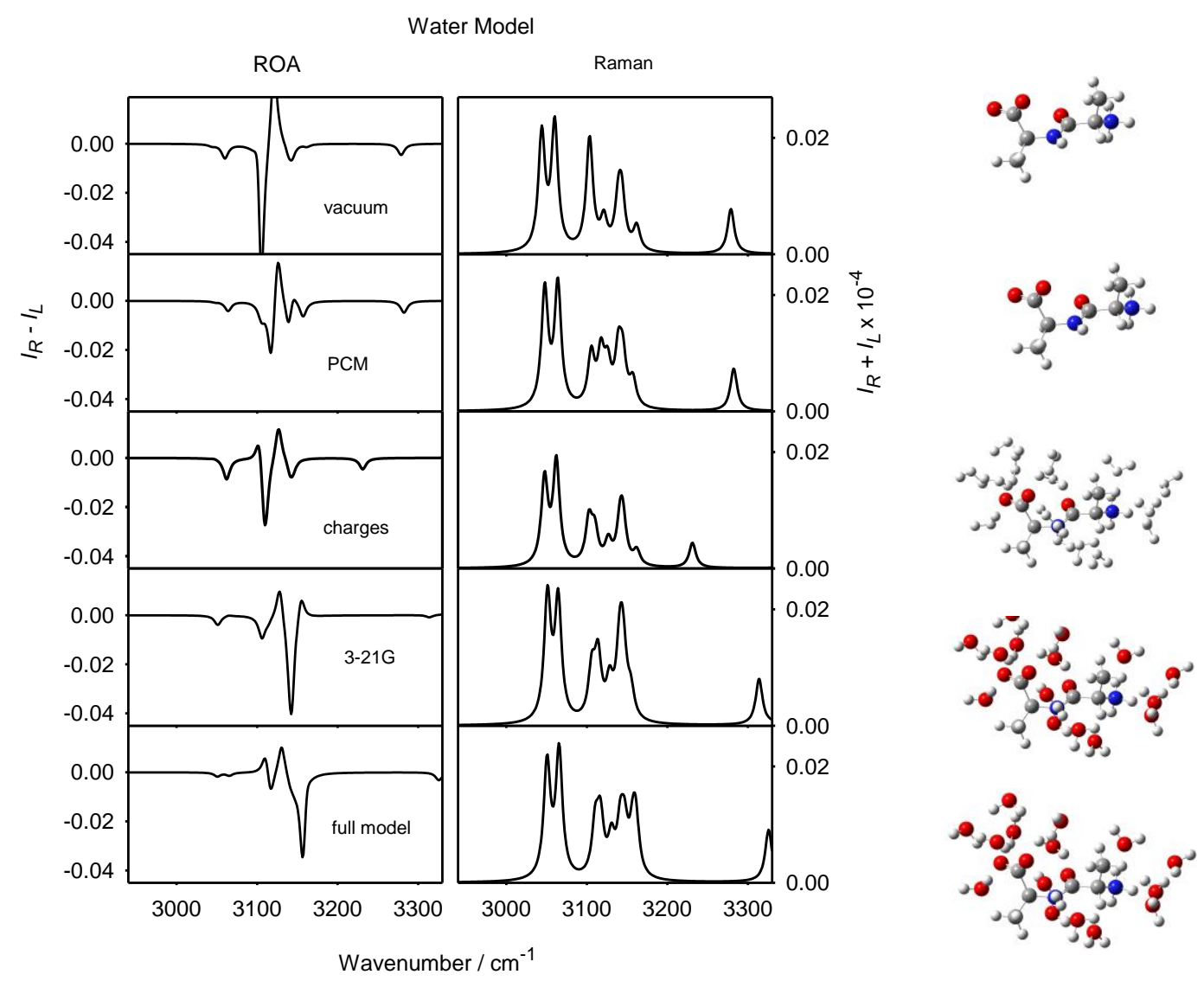

Figure S7. Randomly chosen MD Ala-Ala zwitterion cluster, ROA and Raman spectra, (from bottom to the top:) calculated for a full cluster (B3PW91/6-311++G**/PCM), with a smaller 3-21G basis set on waters, charges instead of water atoms, PCM only, and Ala-Ala in vacuum. 
Protonated AlaAla
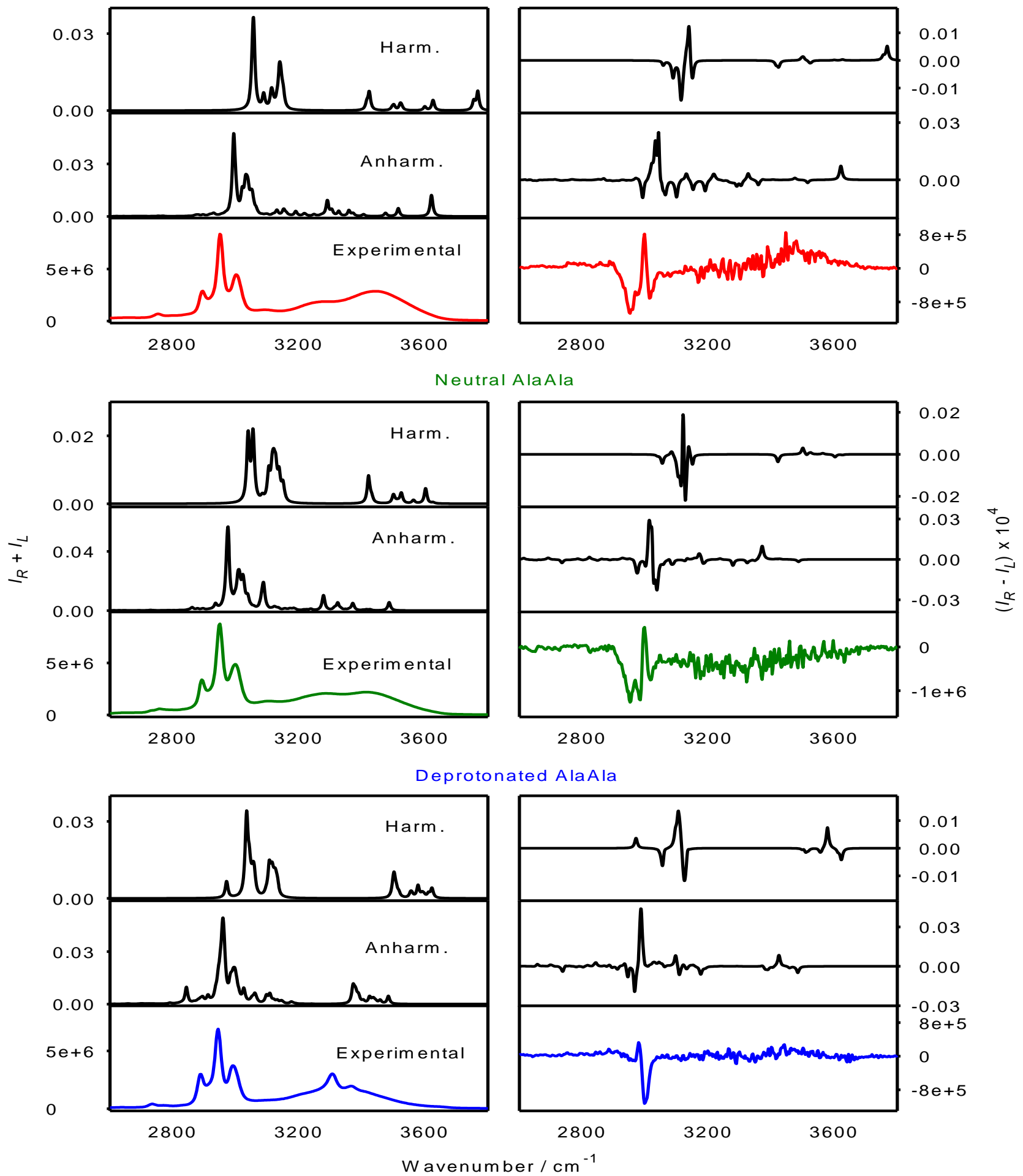

Figure S8. Simulated Raman and ROA spectra for the three Ala-Ala forms at the harmonic and anharmonic/PT2 levels (no MD averaging), and the experiment. 
Protonated AlaAla
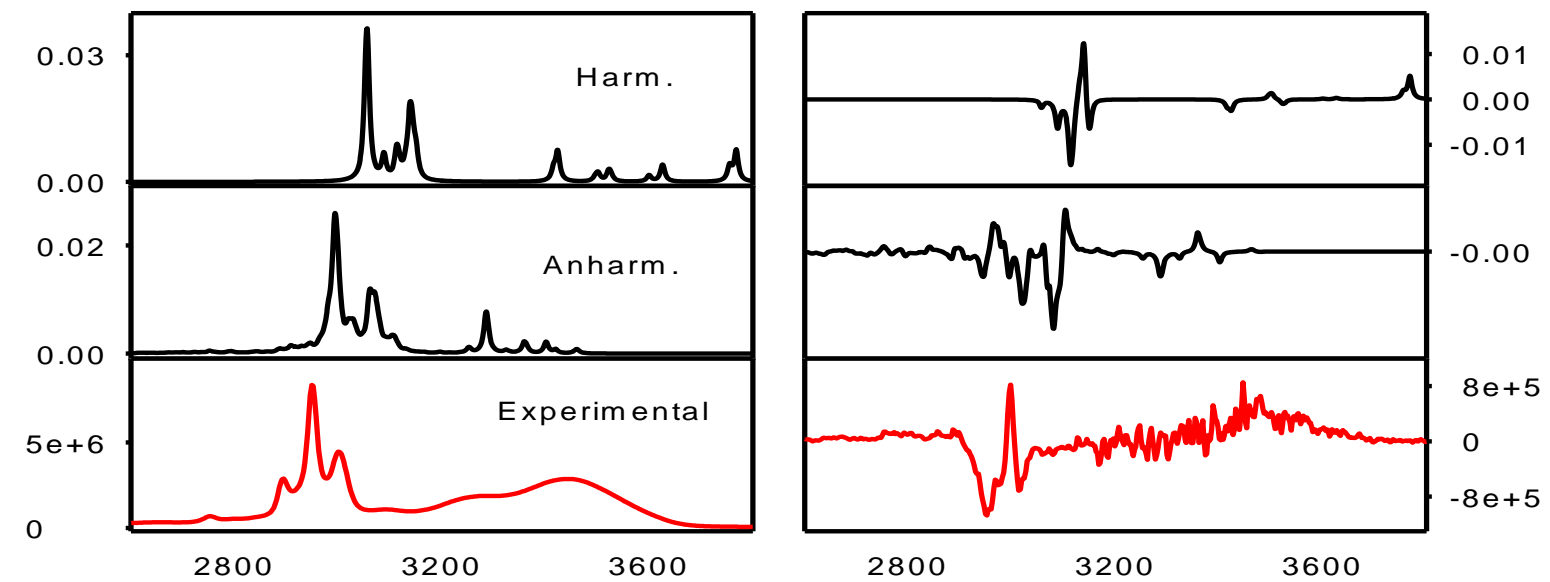

Neutral AlaAla
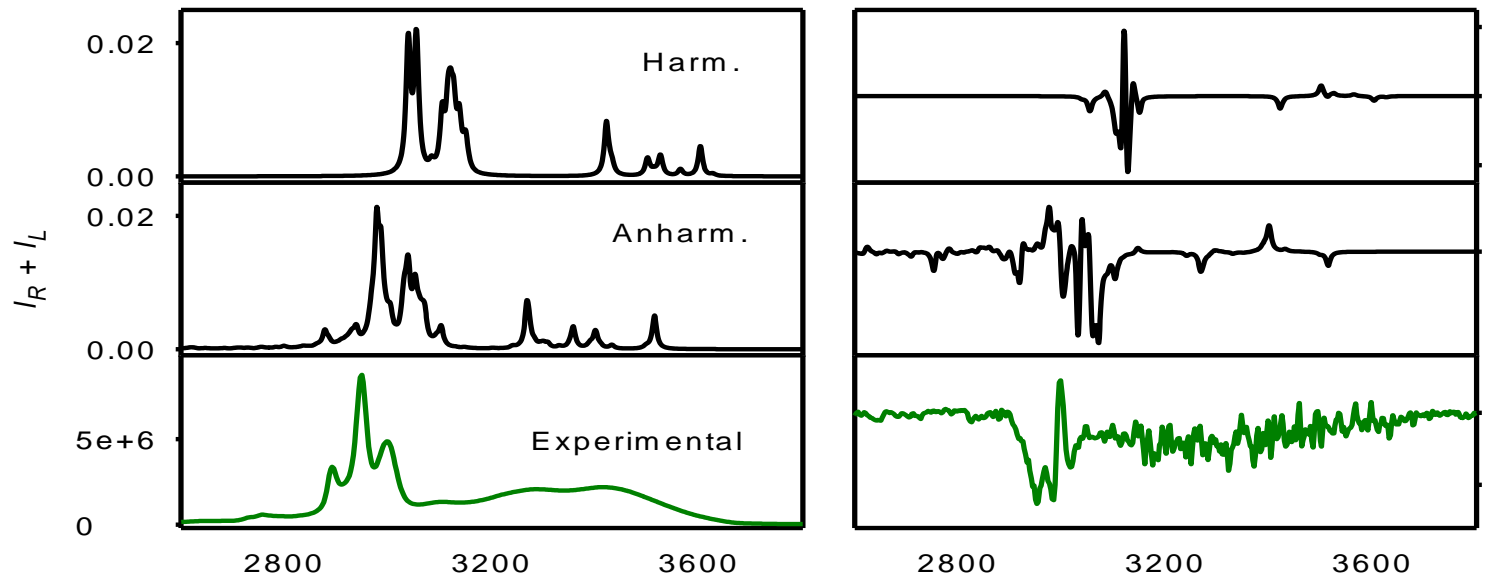

0.02

0.00

$-0.02$

+
$\stackrel{0}{x}$
$=$
$=$
$=$

Deprotonated AlaAla
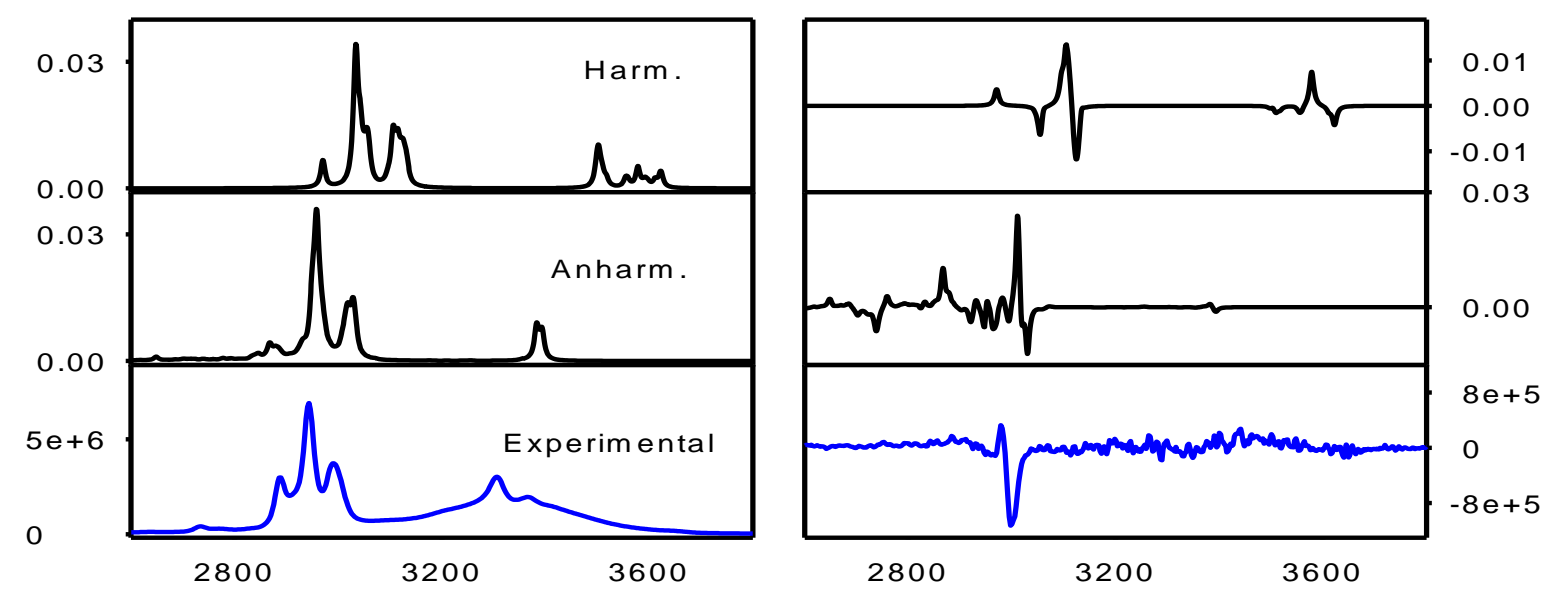

Wavenumber $/ \mathrm{cm}^{-1}$

Figure S9. Simulated Raman and ROA spectra for the three Ala-Ala forms at the harmonic and anharmonic/LVCI levels (no MD averaging), and the experiment. 
Table S1. Positions $\left(\mathrm{cm}^{-1}\right)$ and ROA/Raman Ratios $(C I D)$ of the Main Experimental Bands

\begin{tabular}{cccc}
\hline & Raman & Roa & $C I D \times 10^{5}$ \\
\hline Protonated & 2754 & - & - \\
& 2895 & - & - \\
& 2950 & 2951 & -1.2 \\
& - & 2976 & -2.2 \\
& 3001 & 2997 & 1.8 \\
& - & 3013 & -2.2 \\
& & & \\
Zwitterion & 2759 & - & - \\
& 2893 & - & - \\
& 2949 & 2952 & -1.2 \\
& - & 2984 & -2.9 \\
& 2998 & 2998 & 0.8 \\
& & 3016 & -2.5 \\
& & & \\
Protonated & 2735 & - & - \\
& 2889 & - & - \\
& 2943 & 2949 & -0.1 \\
& - & 2979 & 1.1 \\
& 2991 & 2997 & -3.3 \\
\hline
\end{tabular}


Table S2. Ala-Ala, Principal Torsion Angles, Fully Optimized (B3PW91/6-311++G**/PCM) and average MD values

\begin{tabular}{ccccc}
\hline & A & B & C & D \\
\hline Protonated-MD & & & & \\
\hline$\varphi$ & -154 & -71 & -155 & -73 \\
\hline$\psi$ & 153 & 157 & -57 & -58 \\
\hline Protonated-PCM & & & & \\
\hline$\varphi$ & -153 & -103 & -149 & $-156^{*}$ \\
\hline$\psi$ & 159 & 157 & -56 & $-36^{*}$ \\
\hline Zwitterion-MD & & & & \\
\hline$\varphi$ & -155 & -73 & -157 & -69 \\
$\psi$ & 152 & 152 & -74 & -64 \\
\hline$\psi$ & & & & \\
\hline$\Psi$ Zwitterion-PCM & -160 & $-160^{*}$ & $-162^{*}$ & $-162^{*}$ \\
\hline$\varphi$ & 162 & $174^{*}$ & $-175^{*}$ & $-20^{*}$ \\
\hline$\psi$ & & & \\
\hline Deprotonated-MD & -150 & -60 & -151 & -59 \\
\hline & 113 & 94 & -5 & -1 \\
\hline Deprotonated-PCM & & & \\
\hline$\varphi$ & $-159^{*}$ & $-157^{*}$ & -154 & $-155^{*}$ \\
\hline & $-153^{*}$ & $137^{*}$ & 7 & $8^{*}$
\end{tabular}

Asterisk (*) means that the structure did not optimize to the initial conformer (A, B, C, D). 
Table S3. AlaAla, Fundamental Modes from B3PW91/6-311++G**/PCM Computations on the Most Populated Conformers

\begin{tabular}{|c|c|c|c|c|c|c|}
\hline \multicolumn{3}{|c|}{ Protonated } & \multirow{2}{*}{$\frac{\text { Zwitterion }}{-}$} & \multicolumn{3}{|c|}{ Deprotonated } \\
\hline $\mathrm{v}_{\mathrm{OH}}$ & 3768 & $v(\mathrm{OH})$ & & & - & \\
\hline$v_{\mathrm{NH}}$ & 3626 & amide $\mathrm{A}$ & 3602 & amide $\mathrm{A}$ & 3605 & amide $\mathrm{A}$ \\
\hline$v_{\mathrm{NH}}$ & 3522 & $\mathrm{NH}_{3}$ as & 3525 & $\mathrm{NH}_{3}$ as & 3578 & $\mathrm{NH}_{2}$ as \\
\hline$v_{\mathrm{NH}}$ & 3501 & $\mathrm{NH}_{3}$ as & 3500 & $\mathrm{NH}_{3}$ as & 3499 & $\mathrm{NH}_{2} \mathrm{~s}$ \\
\hline$v_{\mathrm{NH}}$ & 3424 & $\mathrm{NH}_{3} \mathrm{~S}$ & 3421 & $\mathrm{NH}_{3} \mathrm{~s}$ & - & \\
\hline$v_{\mathrm{CH}}$ & 3150 & $\mathrm{Me}$ a $(\mathrm{N})$ & 3150 & $\mathrm{Me} a(N)$ & 3122 & $\operatorname{Me~a~}(\mathrm{C}),{ }^{\alpha} \mathrm{C}(\mathrm{C})$ \\
\hline$v_{\mathrm{CH}}$ & 3143 & $\mathrm{Me} \mathrm{a}(\mathrm{C})$ & 3137 & $\operatorname{Me~a~}(\mathrm{N}),{ }^{\alpha} \mathrm{C}(\mathrm{N})$ & 3116 & $\mathrm{Me}, \mathrm{a}(\mathrm{N})$ \\
\hline$v_{\mathrm{CH}}$ & 3139 & Me a $(\mathrm{N}),{ }^{\alpha} \mathrm{C}(\mathrm{N})$ & 3126 & $\operatorname{Me~a~}(\mathrm{C}),{ }^{\alpha} \mathrm{C}(\mathrm{C})$ & 3115 & $\mathrm{Me}$ a $(\mathrm{C})$ \\
\hline$v_{\mathrm{CH}}$ & 3137 & $\operatorname{Me~a~}(\mathrm{C}),{ }^{\alpha} \mathrm{C}(\mathrm{C})$ & 3120 & $\operatorname{Me~a~}(\mathrm{C}),{ }^{\alpha} \mathrm{C}(\mathrm{C})$ & 3106 & $\mathrm{Me}$ a $(\mathrm{N})$ \\
\hline$v_{\mathrm{CH}}$ & 3113 & ${ }^{\alpha} \mathrm{C}(\mathrm{N})$ & 3115 & ${ }^{\alpha} \mathrm{C}(\mathrm{N})$ & 3103 & Me a $(\mathrm{C}),{ }^{\alpha} \mathrm{C}(\mathrm{C})$ \\
\hline$v_{\mathrm{CH}}$ & 3088 & ${ }^{\alpha} \mathrm{C}(\mathrm{C})$ & 3104 & $\mathrm{Me} \mathrm{a}(\mathrm{C}),{ }^{\alpha} \mathrm{C}(\mathrm{N})$ & 3057 & ${ }^{\alpha} \mathrm{C}(\mathrm{N})$ \\
\hline$v_{\mathrm{CH}}$ & 3057 & $\operatorname{Me} s(N)$ & 3054 & $\operatorname{Me} s(N)$ & 3035 & $\operatorname{Me~s}(\mathrm{C})$ \\
\hline$v_{\mathrm{CH}}$ & 3054 & $\operatorname{Me~s}(\mathrm{C})$ & 3038 & $\operatorname{Me~s}(\mathrm{C})$ & 3031 & $\operatorname{Me~} s(\mathrm{~N})$ \\
\hline$v_{\mathrm{C}=\mathrm{O}}$ & 1803 & $\mathrm{COOH}$ & - & & - & \\
\hline$v_{\mathrm{C}=\mathrm{O}}$ & 1729 & amide I & 1723 & amide I & 1689 & amide I \\
\hline$\delta_{\mathrm{NH}}$ & 1661 & $\mathrm{NH}_{3} \mathrm{a}$ & 1665 & $\mathrm{NH}_{3} \mathrm{a}$ & 1622 & $\mathrm{NH}_{2}$ \\
\hline$\delta_{\mathrm{NH}}$ & 1629 & $\mathrm{NH}_{3} \mathrm{a}$ & 1642 & $\mathrm{NH}_{3} \mathrm{a}$ & 1619 & $\mathrm{NH}_{2}, \mathrm{COO} \mathrm{a}$ \\
\hline$v_{\mathrm{C}=\mathrm{O}}$ & - & & 1634 & $\mathrm{COO}, \mathrm{a}$ & - & \\
\hline$v_{\mathrm{C}-\mathrm{N}}, \delta_{\mathrm{NH}}$ & 1550 & amide II & 1556 & amide II & 1537 & amide II \\
\hline$\delta_{\mathrm{CH}}, \delta_{\mathrm{NH}}$ & 1525 & $\mathrm{NH}_{3} \mathrm{~S}$ & 1519 & $\mathrm{~d}(\mathrm{NH} 3), \mathrm{s}$ & & \\
\hline$\delta_{\mathrm{CH}}$ & 1487 & $\mathrm{Me} a(\mathrm{C})$ & 1488 & $\mathrm{Me}$ a $(\mathrm{N})$ & 1484 & Me a $(\mathrm{N})$ \\
\hline$\delta_{\mathrm{CH}}$ & 1484 & $\mathrm{Me}$ a $(\mathrm{N})$ & 1481 & $\mathrm{Me}$ a $(\mathrm{C})$ & 1474 & $\mathrm{Me}$ a $(\mathrm{C})$ \\
\hline$\delta_{\mathrm{CH}}$ & 1480 & $\mathrm{Me}$ a $(\mathrm{C})$ & 1476 & $\mathrm{Me} a(\mathrm{~N})$ & 1474 & $\mathrm{Me}$ a $(\mathrm{C})$ \\
\hline$\delta_{\mathrm{CH}}$ & 1479 & $\operatorname{Me~a~}(\mathrm{N})$ & 1473 & $\mathrm{Me} a(\mathrm{C})$ & 1470 & $\mathrm{Me} a(\mathrm{~N})$ \\
\hline$\delta_{\mathrm{CH}}$ & 1423 & $\operatorname{Me~s}(\mathrm{C}),{ }^{\alpha} \mathrm{C}(\mathrm{C})$ & 1423 & $\operatorname{Me~s}(\mathrm{N}),{ }^{\alpha} \mathrm{C}(\mathrm{N})$ & 1421 & ${ }^{\alpha} \mathrm{C}(\mathrm{N})$ \\
\hline$\delta_{\mathrm{CH}} v_{\mathrm{C}=\mathrm{O}}$ & 1423 & $\operatorname{Me~s}(\mathrm{N}),{ }^{\alpha} \mathrm{C}(\mathrm{N})$ & 1408 & $\operatorname{Me~s}(\mathrm{N}),{ }^{\alpha} \mathrm{C}(\mathrm{N})$ & 1405 & $\operatorname{Me~s}(\mathrm{C}), \mathrm{COO} \mathrm{s}$ \\
\hline$\delta_{\mathrm{CH}}$ & 1411 & $\operatorname{Me~s}(\mathrm{N}),{ }^{\alpha} \mathrm{C}(\mathrm{N})$ & 1405 & $\operatorname{Me~s}(\mathrm{C}),{ }^{\alpha} \mathrm{C}(\mathrm{C})$ & 1384 & $\operatorname{Me~s}(\mathrm{N})$ \\
\hline$\delta_{\mathrm{CH}}$ & 1397 & $\operatorname{Me~s}(\mathrm{C})$ & 1373 & $\operatorname{Me~s}(\mathrm{C})$ & 1369 & $\operatorname{Me~s}(\mathrm{C})$ \\
\hline$\delta_{\mathrm{CH}}$ & 1357 & ${ }^{\alpha} \mathrm{C}(\mathrm{N}),{ }^{\alpha} \mathrm{C}(\mathrm{C})$ & 1358 & ${ }^{\alpha} \mathrm{C}(\mathrm{N}),{ }^{\alpha} \mathrm{C}(\mathrm{C})$ & 1364 & ${ }^{\alpha} \mathrm{C}(\mathrm{N}),{ }^{\alpha} \mathrm{C}(\mathrm{C})$ \\
\hline$\delta_{\mathrm{CH}}$ & 1338 & ${ }^{\alpha} \mathrm{C}(\mathrm{N}),{ }^{\alpha} \mathrm{C}(\mathrm{C})$ & 1334 & ${ }^{\alpha} \mathrm{C}(\mathrm{N}),{ }^{\alpha} \mathrm{C}(\mathrm{C})$ & 1346 & ${ }^{\alpha} \mathrm{C}(\mathrm{N}),{ }^{\alpha} \mathrm{C}(\mathrm{C})$ \\
\hline$\delta_{\mathrm{XH}}$ & 1292 & $\mathrm{OH}$ & 1280 & ${ }^{\alpha} \mathrm{C}(\mathrm{C})$ & 1285 & ${ }^{\alpha} \mathrm{C}(\mathrm{C})$ \\
\hline$\delta_{\mathrm{NH}}$ & 1263 & $\mathrm{NH}$ & 1265 & $\mathrm{NH}$ & 1244 & $\mathrm{NH}$ \\
\hline$\delta_{\mathrm{CH}}$ & 1222 & $\mathrm{NH}_{3}$, Me wag $(\mathrm{N})$ & 1224 & $\mathrm{NH}_{3}, \mathrm{Me}$ wag $(\mathrm{N})$ & 1206 & $\mathrm{NH}$ \\
\hline$\delta_{\mathrm{CH}}$ & 1201 & Me wag (C) & 1169 & Me wag (C) & 1159 & ${ }^{\alpha} \mathrm{C}(\mathrm{N})$ \\
\hline$\delta_{\mathrm{OH}}$ & 1151 & & - & & 1146 & Me wag (C) \\
\hline
\end{tabular}

$v$ - stretching, $\delta$ - bending, s - symmetric, as - asymmetric, $(\mathrm{C})$ and $(\mathrm{N})$ indicate the $\mathrm{C}$ and $\mathrm{N}$ terminus, respectively, Me - methyl, wag - wagging/rocking of all groups 


\section{References}

(1) Burke, K.; Perdew, J. P.; Wang, Y. Electronic Density Functional Theory: Recent Progress and New Directions; Plenum: New York, 1998.

(2) Becke, A. Density-Functional Exchange-Energy Approximation with Correct Asymptotic Behavior. Phys. Rev. A 1988, 38, 3098-3100.

(3) Perdew, J. P.; Chevary, J. A.; Vosko, S. H.; Jackson, K. A.; Pederson, M. R.; Singh, D. J.; Fiolhais, C. Atoms, Molecules, Solids, and Surfaces: Applications of the Generalized Gradient Approximation for Exchange and Correlation. Phys. Rev. B 1992, 46, 6671-6687.

(4) Perdew, J. P. Density-Functional Approximation for the Correlation Energy of the Inhomogeneous Electron Gas. Phys. Rev. B 1986, 33, 8822-8824.

(5) Zhao, Y.; Truhlar, D. G. Density Functional for Spectroscopy: No Long-Range SelfInteraction Error, Good Performance for Rydberg and Charge-Transfer States, and Better Performance on Average Than B3lyp for Ground States. J. Phys. Chem. A 2006, 110, 13126-13130.

(6) Becke, A. D. Density-Functional Thermochemistry. Iii. The Role of Exact Exchange. J. Chem. Phys. 1993, 98, 5648-5652.

(7) Yanai, T.; Tew, D.; Handy, N. C. A New Hybrid Exchange-Correlation Functional Using the Coulomb-Attenuating Method (Cam-B3lyp). Chem. Phys. Lett. 2004, 393, 51-57.

(8) Zhao, Y.; Truhlar, D. G. The M06 Suite of Density Functionals for Main Group Thermochemistry, Thermochemical Kinetics, Noncovalent Interactions, Excited States, and Transition Elements: Two New Functionals and Systematic Testing of Four M06-Class Functionals and 12 Other Functionals. Theor. Chem. Acc. 2008, 120, 215-241.

(9) Heyd, J.; Scuseria, G. Efficient Hybrid Density Functional Calculations in Solids: The Hs-Ernzerhof Screened Coulomb Hybrid Functional. J. Chem. Phys. 2004, 121 1187-1192.

(10) Boese, A. D.; Martin, M. L. Development of Density Functionals for Thermochemical Kinetics. J. Chem. Phys. 2004, 121 3405-3416.

(11) Austin, A.; Petersson, G.; Frisch, M. J.; Dobek, F. J.; Scalmani, G.; Throssell, K. A Density Functional with Spherical Atom Dispersion Terms. J. Chem. Theory and Comput. 2012, 8, 4989.

(12) Møller, C.; Plesset, M. S. Note on an Approximation Treatment for Many-Electron Systems. Phys. Rev. 1934, 46, 618-622. 